\title{
Influence of pump power and modulation instability gain spectrum on seeded supercontinuum and rogue wave generation
}

Sørensen, Simon Toft; Larsen, Casper; Møller, Uffe; Moselund, M.; Thomsen, Carsten L.; Bang, Ole

Published in:

Optical Society of America. Journal B: Optical Physics

Link to article, DOI:

10.1364/JOSAB.29.002875

Publication date:

2012

Document Version

Publisher's PDF, also known as Version of record

Link back to DTU Orbit

Citation (APA):

Sørensen, S. T., Larsen, C., Møller, U., Moselund, M., Thomsen, C. L., \& Bang, O. (2012). Influence of pump power and modulation instability gain spectrum on seeded supercontinuum and rogue wave generation. Optical Society of America. Journal B: Optical Physics, 29(10), 2875-2885. https://doi.org/10.1364/JOSAB.29.002875

\section{General rights}

Copyright and moral rights for the publications made accessible in the public portal are retained by the authors and/or other copyright owners and it is a condition of accessing publications that users recognise and abide by the legal requirements associated with these rights.

- Users may download and print one copy of any publication from the public portal for the purpose of private study or research.

- You may not further distribute the material or use it for any profit-making activity or commercial gain

- You may freely distribute the URL identifying the publication in the public portal 


\title{
Influence of pump power and modulation instability gain spectrum on seeded supercontinuum and rogue wave generation
}

\author{
Simon Toft Sørensen, ${ }^{1, *}$ Casper Larsen, ${ }^{1}$ Uffe Møller, ${ }^{1}$ Peter M. Moselund, ${ }^{2}$ \\ Carsten L. Thomsen, ${ }^{2}$ and Ole Bang ${ }^{1,2}$ \\ ${ }^{1}$ DTU Fotonik, Department of Photonics Engineering, Technical University of Denmark, 2800 Kgs. Lyngby, Denmark \\ ${ }^{2}$ NKT Photonics A/S, Blokken 84, DK-3460, Birkerød, Denmark \\ *Corresponding author: stso@fotonik.dtu.dk
}

Received April 5, 2012; revised August 23, 2012; accepted August 28, 2012;

posted August 28, 2012 (Doc. ID 166112); published September 21, 2012

\begin{abstract}
The noise properties of a supercontiuum can be significantly improved both in terms of coherence and intensity stability by modulating the input pulse with a seed. In this paper, we numerically investigate the influence of the seed wavelength, the pump power, and the modulation instability gain spectrum on the seeding process. The results can be clearly divided into a number of distinct dynamical regimes depending on the initial four-wave mixing process. We further demonstrate that seeding can be used to generate coherent and incoherent rogue waves, depending on the modulation instability gain spectrum. Finally, we show that the coherent pulse breakup afforded by seeding is washed out by turbulent solitonic dynamics when the pump power is increased to the kilowatt level. Thus our results show that seeding cannot improve the noise performance of a high power supercontinuum source. () 2012 Optical Society of America
\end{abstract} OCIS codes: $\quad 030.1640,190.4370,190.4380,320.6629$

\section{INTRODUCTION}

Supercontinuum (SC) sources have by now been established as a new type of light source well suited for many characterization and imaging applications [1]. Many of these applications require spectra with high power density in the visible part of the spectrum, which can be achieved in nonlinear fibers with a group-velocity (GV) profile that allows GV matching between long wavelength solitons and dispersive waves at visible wavelengths [2]. The concept of GV matching has been used to show how the spectra can be extended into the deepblue by optimizing the fiber structure [3], by tapering the fiber [4-6], or by doping the fiber [7]. Other ways of pushing the spectrum toward the blue include seeding with the second harmonic [8] , using concatenated fibers [9] , or back-seeding part of the SC [10-12].

Today's commercial SC sources are using high-power picosecond (ps) or nanosecond (ns) pump lasers, where the SC generation is initiated by unseeded modulational instability (MI). Because of the high stability of the pump laser the main source of shot-to-shot noise stems from the fact that unseeded MI grows from noise. Subsequently, MI leads to a pulse breakup, which generates a distributed spectrum of solitons that interact and transfer energy between each other during collisions [13]. The interaction will add to the noise, because it depends strongly on the relative phase and amplitude of the solitons, but on average there is a preferential transfer of energy from the smaller to the larger solitons [14-17]. This energy transfer can lead to the formation of rare large amplitude solitons, also known as rogue waves in optics [18] or highly localized modes in biophysics [14].

To reduce the noise it has been proposed to provide a seed, i.e., a weak pulse with a frequency offset relative to the pump, within the MI gain spectrum in order to ensure a deterministic rather than noise-seeded pulse breakup [13,19-24]. In particular, Genty et al. [20] numerically investigated seeding with different seed frequencies in a silica fiber with an MI gain peak at $8 \mathrm{THz}$ and showed that a seed at $5 \mathrm{THz}$ from the pump, i.e., at approximately two-thirds of the MI gain peak, gave an optimum improvement of the broadening and stability of the SC. In all cases a low pump peak power of $75 \mathrm{~W}$ (pulse energy $0.4 \mathrm{~nJ}$ ) was used, which was then split between the pump and seed. In [21], a coherent comb-like SC was generated by seeding a high peak power $(10 \mathrm{~kW})$ ps pulse, and an optimal fiber length was determined, for which the comb remains coherent. The optimum length was found to be 5-10 cm for typical commercial SC sources; broad spectra without comb structure requires longer fiber lengths and was hence not investigated. Li et al. [24] investigated the influence of a weak CW seed on low power SC generation in a dispersion-shifted fiber, and described how seeding leads to a pulse breakup caused by four-wave mixing (FWM).

Experimentally, seeded SC generation was investigated in $[\underline{10}, \underline{12}, \underline{19}, \underline{22}, \underline{23}]$. In $[19,22,23]$ the SC generation was induced by triggering a sub-threshold pump with a seed pulse or continuous wave. Improved spectral stability and coherence was demonstrated in [19], and in [22,23] an optimum spectral broadening was found when seeding near the MI gain peak. An enhancement of the spectral bandwidth with increasing pump or seed power was further demonstrated in [22], but only for low power near the SC threshold where the spectral broadening is caused by a single soliton generated from the seeding process. The results presented in $[19,22,23]$ are different from the optimum conditions found in $[20, \overline{24}]$ and here, where the pulse break-up is caused by the amplification of 
a FWM cascade. A different approach was pursued in [미, $\underline{12}]$, where a fraction of the generated SC from one pulse was used as a seed for the following pump pulse. This, however, is fundamentally different from modulating the pump pulse with a seed as in this work and [19-24].

While it has thus been shown that seeding can reduce the noise of an SC, this has been either at low pump power, often close to the MI threshold, or for very short fibers. The previous investigations are thus far from commercial SC sources with spectra extending down in the visible, which are typically pumped using ns or ps pump pulses at $1064 \mathrm{~nm}$ with high peak powers in the order of $\sim 10 \mathrm{~kW}$ and fiber lengths of $\sim 10 \mathrm{~m}$. Here we investigate the effect of seeding under a variety of conditions, and explain what happens as we approach the parameters of a commercial SC source. In particular, we investigate the influence of the seed wavelength and MI gain spectrum on seeding at various power levels above the SC threshold, from which we highlight a number of distinct dynamical regimes. We further demonstrate for the first time how seeding leads to the generation of coherent or incoherent rogue solitons depending on the MI spectrum. Finally, we explain how the coherent pulse breakup caused by the seeding is eventually washed out by turbulent solitonic dynamics when the peak power is increased to the kilowatt $(\mathrm{kW})$ level.

This paper is structured as follows. In Sections $\underline{2}$ and $\underline{3}$, we explain how the MI gain spectrum can be calculated and altered, and how the statistical properties of an SC can be analyzed both in terms of coherence and intensity stability. In Section 4 we proceed to analyze the effects of seeding at a relatively low peak power of $250 \mathrm{~W}$ to illustrate the general improvements in coherence and intensity stability, and explain the influence of the MI gain spectrum on seeding. We further describe how this can be used to generate a coherent or incoherent rogue soliton. Finally, in Section $\underline{5}$ we investigate the effects of seeding at higher peak powers.

\section{MODULATIONAL INSTABILITY GAIN AND SEEDING}

The MI gain spectrum $g(\Omega)$, taking into account the Raman response function, is given by (see, e.g., [25])

$$
g(\Omega)=\operatorname{Im}\left\{\Delta k_{o} \pm \sqrt{\left(\Delta k_{e}+2 \gamma P_{0} \tilde{R}(\Omega)\right) \Delta k_{e}}\right\}
$$

where $\Omega$ is the angular frequency offset relative to the pump. $\Delta k_{o}$ and $\Delta k_{e}$ are sums over odd and even order derivatives of the propagation constant $\beta$,

$$
\Delta k_{o}=\sum_{m=1}^{\infty} \frac{\bar{\beta}_{2 m+1}}{(2 m+1) !} \Omega^{2 m+1}, \quad \Delta k_{e}=\sum_{m=1}^{\infty} \frac{\bar{\beta}_{2 m}}{2 m !} \Omega^{2 m}
$$

where $\bar{\beta}_{m}=\partial^{m} \beta /\left.\partial \Omega^{m}\right|_{\Omega=0} \cdot \gamma$ is the nonlinear parameter, $P_{0}$ is the peak power, and $\tilde{R}(\Omega)$ is the Raman response for silica, which can be approximated by [26]

$$
\tilde{R}(\Omega)=\left(1-f_{R}\right)+f_{R} \frac{\tau_{1}^{2}+\tau_{2}^{2}}{\tau_{2}^{2}-\tau_{1}^{2}\left(i+\tau_{2} \Omega\right)^{2}},
$$

where $f_{R}=0.18$ is the fractional contribution of the Raman response, $\tau_{1}=12.2$ fs and $\tau_{2}=32$ fs.
The MI gain is strongly influenced by the Raman effect, and in order to make a detailed investigation of the influence of the MI gain spectrum on seeding under a variety of conditions, we wanted the possibility of tuning the peak of the MI gain spectrum from significantly below to significantly above the peak of the Raman gain spectrum at $13.2 \mathrm{THz}$ (in silica). For a given fiber this can be achieved by changing either the pump wavelength or peak power. In this work we use a solid core silica photonic crystal fiber (PCF) with pitch (hole spacing) $\Lambda=3.6 \mu \mathrm{m}$ and hole-to-pitch ratio $d / \Lambda=0.52$, resulting in a zero-dispersion wavelength (ZDW) at $1054.2 \mathrm{~nm}$. Pump pulses with a temporal width (FWHM) of $3 \mathrm{ps}\left(T_{0} \approx 3 \mathrm{ps} /\right.$ 1.665 for a Gaussian pulse) and a fiber length of $10 \mathrm{~m}$ were used in all simulations presented in this paper. The dispersion and effective area are shown in Fig. 1(a), whereas Figs. 1(b) -1 (c) show how the MI gain spectrum changes with wavelength and peak power, respectively. For this particular fiber the peak of the MI spectrum can thus easily be swept over the desired range by simply changing the wavelength or peak power of the pump over a range that is experimentally realisable. The frequency of the MI gain maximum increases, i.e., moves away from the pump, when the pump wavelength is decreased or the peak power is increased. We stress that although the pump powers in Figs. $1(\mathrm{~b})-1(\mathrm{c})$ are all significantly lower than the $\sim 10 \mathrm{~kW}$ used in high-power commercial $\mathrm{SC}$ sources, it is well above the threshold for SC generation and the power levels previously reported in the literature on seeding in, e.g., [19,20,22-24]. As mentioned earlier, seeding at high-power was investigated in [21] but only for very short fiber lengths.

Figure 1(d) shows the walk-off length of the pump and seed and MI gain length, respectively. The walk-off length is the propagation distance over which the two pulses are separated by less than the pulse width, i.e., $T_{0} /\left|v_{g \text {,pump }}^{-1}-v_{g \text {,seed }}^{-1}\right|$, where $v_{g, i}$ is the group-velocity. When the walk-off length is shorter than the MI gain length, the seed cannot have an effect. In other words, a seed placed in the tail of the gain spectrum cannot be expected to have any significant effect because of a too short interaction length with the pump.

The work in this paper is based on solutions to the generalized nonlinear Schrödinger equation (GNLSE), which has become the standard for simulating nonlinear pulse propagation in optical fibers due to its ability to reproduce spectra and noise properties in agreement with experiments [1,27]. The GNLSE takes into account the effects of nonlinearities, the delayed Raman response, and higher-order dispersion. We used the implementation of [28] with the GNLSE solved in the interaction-picture [29], and included a noise background of one photon with a random phase in each discretization bin. This allows ensemble statistics to be calculated by carrying out simulations under identical conditions, but with different initial noise. In the simulations we used a Gaussian pump and seed of identical temporal width $T_{0}$. The two pulses were temporally overlapping, and the seed given a frequency offset $\nu_{\text {mod }}$, where $\nu_{\text {mod }}>0$ corresponds to seeding at a wavelength longer than that of the pump, i.e., in the Stokes band. The temporal pulse envelope $A(t)$ was hence,

$$
A(t)=\left(\sqrt{P_{p}}+\sqrt{P_{s}} e^{i 2 \pi \nu_{\text {mod }} t}\right) \exp \left(\frac{-t^{2}}{2 T_{0}^{2}}\right)
$$



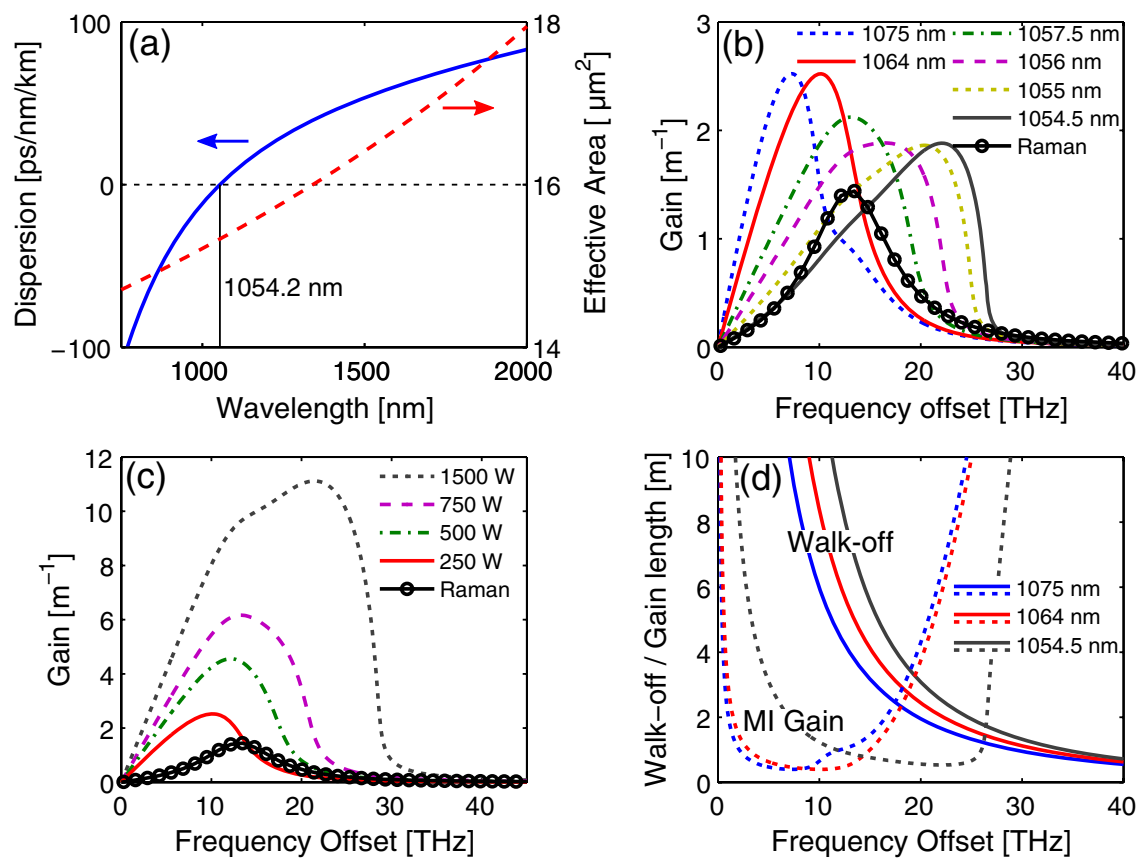

Fig. 1. (Color online) (a) Dispersion and effective area for the used PCF with $\Lambda=3.6 \mu \mathrm{m}$ and $d / \Lambda=0.52$. (b)-(c) MI gain spectra as a function of seed frequency offset relative to the pump for varying pump wavelength (b) and peak power (c). The peak power in (b) is $250 \mathrm{~W}$ and the pump wavelength in (c) is $1064 \mathrm{~nm}$. The Raman gain is shown for comparison. (d) Walk-off length (solid lines) and MI gain length (dotted lines) as a function of frequency offset, calculated for $T_{0}=3 \mathrm{ps} / 1.665$ and a peak power of $250 \mathrm{~W}$.

where $P_{p}$ and $P_{s}$ are the peak powers of the pump and seed, respectively. This is similar to what was used in [20], although in [20] the peak power was shared between the pump and seed as would be the case if the seed is generated from the pump by some frequency shifting technique. This however means that changing the power of the seed also causes a change of the MI gain spectrum. Here we consider a seed that is independent of the pump in order to have the same MI gain spectrum irrespectively of the seed peak power. Using Eq. (4) it is thus straightforward to sweep the pump-seed frequency offset and peak power of the seed for a fixed MI gain spectrum. We emphasize that the narrow-banded pulses used here have a FWHM spectral width of only $0.15 \mathrm{THz}$. The seed therefore only has a substantial spectral overlap with the pump for very small offsets, i.e. $\nu_{\text {mod }} \approx 0 \mathrm{THz}$, which results in an effective increase of the peak power and hence the MI gain spectrum.

\section{QUANTIFYING SUPERCONTINUUM NOISE}

Typically, the noise is quantified by the widely used spectral coherence function calculated as an ensemble average over independent SC spectra, $\tilde{A}_{i}(\omega)$ [30],

$$
\left|g_{12}^{(1)}(\omega)\right|=\left|\frac{\left\langle\tilde{A}_{i}^{*}(\omega) \tilde{A}_{j}(\omega)\right\rangle_{i \neq j}}{\sqrt{\left\langle\left|\tilde{A}_{i}(\omega)\right|^{2}\right\rangle\left\langle\left|\tilde{A}_{j}(\omega)\right|^{2}\right\rangle}}\right|,
$$

where the angle brackets denote ensemble averages and the asterisk denotes complex conjugation. The spectral coherence function provides insight into the stability of an SC and is sensitive to shot-to-shot phase fluctuations. However, high-power and long-pulsed MI-driven SC generation is predominantly incoherent, as we shall see in the following, and we will therefore also consider the intensity stability quantified here by the signal-to-noise ratio (SNR) defined as the ratio of the mean $\mu$ to the standard deviation $\sigma$,

$$
\operatorname{SNR}(\omega)=\frac{\mu(\omega)}{\sigma(\omega)}
$$

The SNR is inversely proportional to the coefficient of variation introduced as an SC noise measure in [31]. The SNR is better suited for highlighting regions of high intensity stability, whereas the coefficient of variation is a good indicator of noisy regions dominated by statistically rare events.

It is illustrative to integrate the spectral coherence to get the spectrally averaged or so-called overall spectral coherence of an SC ensemble [1]],

$$
\left\langle\left|g_{12}^{(1)}\right|\right\rangle=\frac{\int_{0}^{\infty}\left|g_{12}^{(1)}(\omega)\right|\left\langle|\tilde{A}(\omega)|^{2}\right\rangle \mathrm{d} \omega}{\int_{0}^{\infty}\left\langle|\tilde{A}(\omega)|^{2}\right\rangle \mathrm{d} \omega} .
$$

The overall coherence is, like the spectral coherence function itself, bounded by the interval $0 \leq\left\langle\left|g_{12}^{(1)}\right|\right\rangle \leq 1$, and gives a single value measure of the overall coherence of an SC. Similarly, we introduce the overall SNR to quantify the overall intensity stability of an SC,

$$
\langle\mathrm{SNR}\rangle=\frac{\int_{0}^{\infty} \operatorname{SNR}(\omega)\left\langle|\tilde{A}(\omega)|^{2}\right\rangle \mathrm{d} \omega}{\int_{0}^{\infty}\left\langle|\tilde{A}(\omega)|^{2}\right\rangle \mathrm{d} \omega} .
$$

It should be noted that the overall SNR has no upper boundary.

The statistical properties of the SC are calculated in each numerical discretization bin across the spectrum. For each set of parameters we carried out 200 simulations to calculate the 
statistics of the generated SC. However, for the higher peak powers in Section 5 only 100 simulations were carried out for each set of parameters. This was in all cases found to be sufficient to get consistent results under the conditions considered here, which was checked by using 1000 simulations in selected cases.

\section{SEEDING AT LOW PEAK POWER}

\section{A. Single Shot Dynamics}

We start our analysis by a detailed discussion of seeding a low peak power pump. The reason for this is both to demonstrate the utility of the noise measures introduced in the previous section, but also to highlight a number of general regimes as a reference for the further analysis. To this end, we show in Figs. 2(a)-2(e) the spectral evolution of single simulations for selected pump-seed frequency offsets for a $1055 \mathrm{~nm}$ pump with $P_{p}=250 \mathrm{~W}$ and a seed with $P_{s}=5 \% P_{p}$. In all cases we also show the coherence and SNR calculated over the ensemble of 200 simulations. The white lines mark the width of the MI gain spectrum calculated for the local pump peak power. We define the MI gain width as the region where the gain is larger than $5 \%$ of the maximum MI gain, but show only the upper gain limit and not the one for the low gain region close to the pump. We start the analysis with a pump wavelength of $1055 \mathrm{~nm}$ because pumping close to the ZDW shifts the MI gain peak far away from the pump and above the Raman gain peak, which, as we shall see, yields the richest dynamics. Figure 2(f) shows the MI gain spectrum of the undepleted pump.

Figure 2(a) shows the evolution for zero frequency offset, i.e., overlapping pump and seed, and the results are as expected for unseeded SC generation: The spectral broadening is initiated by noise-seeded MI, which manifests itself as a single set of sidebands positioned at the peaks of the calculated MI gain spectrum. This is followed by the onset of soliton and DW generation from around $5 \mathrm{~m}$. The resulting statistics show only coherence near the residual pump and a flat near-unity (a) $0 \mathrm{THz}$

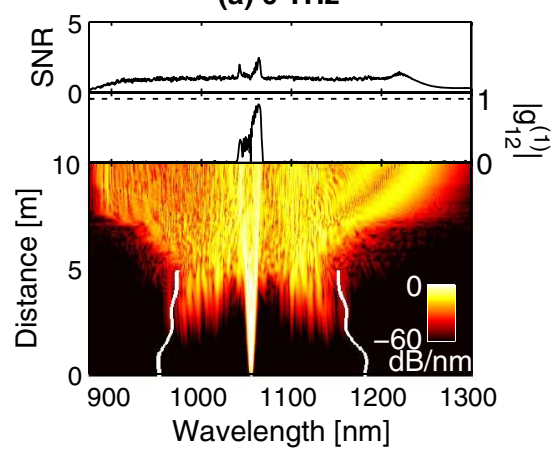

(c) $13 \mathrm{THz}$

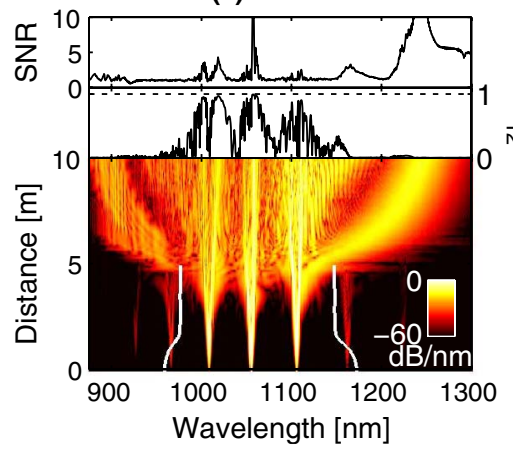

(e) $30 \mathrm{THz}$

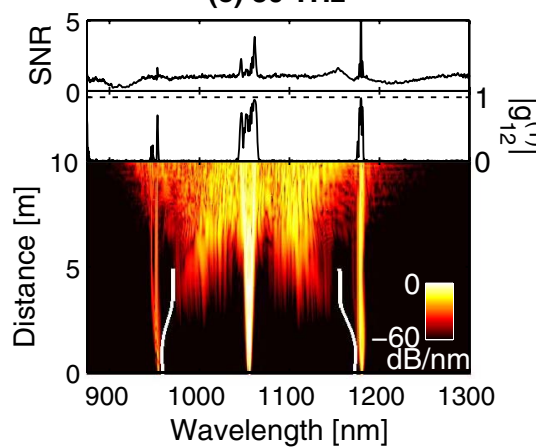

(b) 3 THz

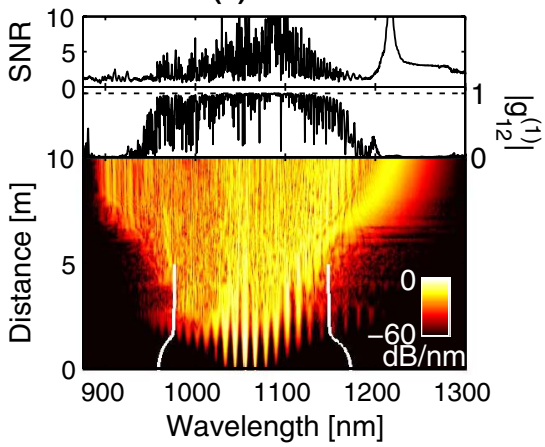

(d) $20 \mathrm{THz}$

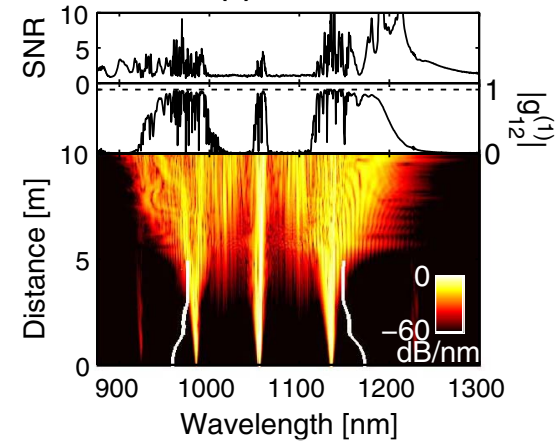

(f)

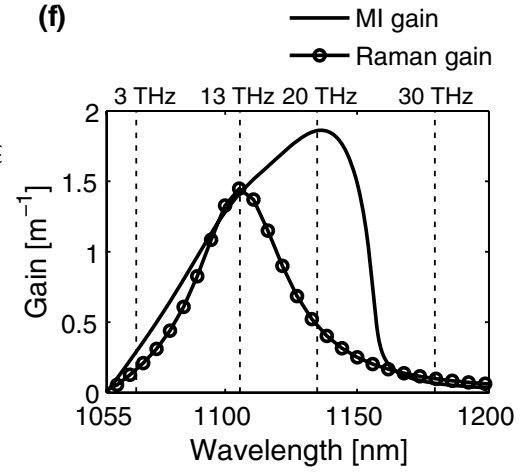

Fig. 2. (Color online) Single-shot simulations of pumping at $1055 \mathrm{~nm}$ with a $250 \mathrm{~W}$ pump and a $5 \%$ seed at frequency offsets of (a)-(e) $0,3,13,20$, and $30 \mathrm{THz}$, respectively. The white lines indicate the MI gain bandwidth. The top rows in (a)-(e) show the ensemble calculated signal-to-noise ratio (SNR) and spectral coherence $\left(\left|g_{12}^{(12)}\right|\right)$. (f) MI and Raman gain curves, the vertical lines correspond to the frequency offsets used in (a)-(e). The frequency offset of $13 \mathrm{THz}(\mathrm{c})$ is the Raman gain peak and $20 \mathrm{THz}(\mathrm{d})$ is the MI gain peak. 
SNR over the entire spectral bandwidth, except near the pump and the soliton at $\sim 1225 \mathrm{~nm}$. We emphasize that the $\mathrm{MI}$ gain spectrum in (f) is calculated for the peak power of the pump alone. The extra peak power added by the overlapping seed in (a) causes a slight shift of the spectrum towards a longer wavelength.

For a small frequency offset of $3 \mathrm{THz}$ in Fig. 2(b), the pulse breakup is initiated by a cascaded FWM process that causes a coherent broadening of the pump. The FWM cascade generates a frequency comb of sidebands with a $3 \mathrm{THz}$ frequency separation. The width of the frequency comb is limited by the width of the MI gain spectrum. It should be noted that the MI gain spectrum is the degenerate FWM gain spectrum of the pump, which amplifies the FWM cascade of the pump and seed. With further propagation a soliton is generated from the FWM process with enough power to redshift outside the MI gain band, with the redshift being enhanced by preferential energy transfer during collisions [14-17]. The output spectrum is coherent over most of the bandwidth, but the soliton at the long wavelength edge of the spectrum has a varying phase from shot to shot, which degrades the coherence at the spectral edges, but leads to a high intensity stability.

In Fig. 2(c) the seed is placed $13 \mathrm{THz}$ from the pump, which is near the peak of the Raman gain. In this case the spectral evolution is dominated by the amplification of a single set of coherent sidebands amplified through degenerate FWM. The second set of sidebands are shifted $26 \mathrm{THz}$ from the pump, which is just on the edge of the initial MI gain spectrum and therefore quickly becomes outside the gain spectrum when the pump depletes. At $\sim 5 \mathrm{~m}$ a massive soliton is ejected from the long wavelength sideband, which is exactly what was referred to as "harnessing and control of optical rogue waves" in [32], where the pump pulse was modulated with a welldefined frequency to eject a large amplitude soliton. The soliton is again not phase-stable from shot to shot, but it is highly intensity-stable. This is opposite to what was reported in [24], where the rogue soliton was coherently generated from a FWM sideband. We will elaborate further on this later in the paper.

In Fig. 2(d) the seed is shifted to the peak of the MI gain at $20 \mathrm{THz}$, which leads to the amplification of a set of well-separated sidebands through FWM. The second FWM lines effectively lie outside the MI gain band. The residual pump and sidebands all undergo SPM and broaden independently of each other. This leads to an output spectrum with three clearly distinct bands with high coherence and SNR. Finally, in Fig. 2(e) the seed is shifted to the tail of the MI gain spectrum, and a single set of sidebands is slowly amplified. The short wavelength nonseeded sideband is entirely generated by FWM and is therefore very weak. The pump is only slightly depleted and experiences noise-seeded MI unaffected by the seed at $1180 \mathrm{~nm}$.

To clarify the influence of the Raman effect on seeding, we show in Fig. 3 the spectral evolution with and without the Raman effect for a seed near the Raman gain peak at $13 \mathrm{THz}$. All other parameters are as in Fig. 2(c). The initial dynamics is similar irrespectively of the Raman effect, but the MI gain bandwidth is reduced much faster without the Raman effect, Eq. (1). When the Raman effect is included, a large soliton is generated from the FWM sideband at $\sim 5 \mathrm{~m}$. The soliton also appears when the Raman effect is turned off, but it is much weaker and does not give rise to any significant intensity stability. This is in good agreement with [17]. In both cases the output spectrum is coherent only near the residual pump and FWM sidebands, resulting in a comparable overall coherence of 0.45 and 0.39 with and without the Raman effect, respectively.

From the above discussion, it is possible to divide the seeding results in Fig. 2 into four distinct regimes depending on the pump-seed frequency offset, $\nu_{\text {mod }}$, and the MI gain bandwidth, $\nu_{\mathrm{MI}}$.

(i) $0<\nu_{\text {mod }} \lesssim \frac{1}{4} \nu_{\mathrm{MI}}$ : A broad FWM cascade gives many bands across the MI gain bandwidth, which leads to a coherent broadening and a spectrum with high coherence and SNR over most of the bandwidth.

(ii) $\frac{1}{4} \nu_{\mathrm{MI}} \lesssim \nu_{\bmod } \lesssim \frac{1}{2} \nu_{\mathrm{MI}}$ : A decreasing number of FWM sidebands are amplified, which diminishes the coherence and SNR improvement.

(iii) $\frac{1}{2} \nu_{\mathrm{MI}} \lesssim \nu_{\text {mod }} \lesssim \nu_{\mathrm{MI}}$ : Amplification of effectively only one set of FWM sidebands, subsequent generation of incoherent large amplitude soliton. Spectrum only coherent near pump and FWM sidebands.

\section{(b) 13 THz, without Raman effect}
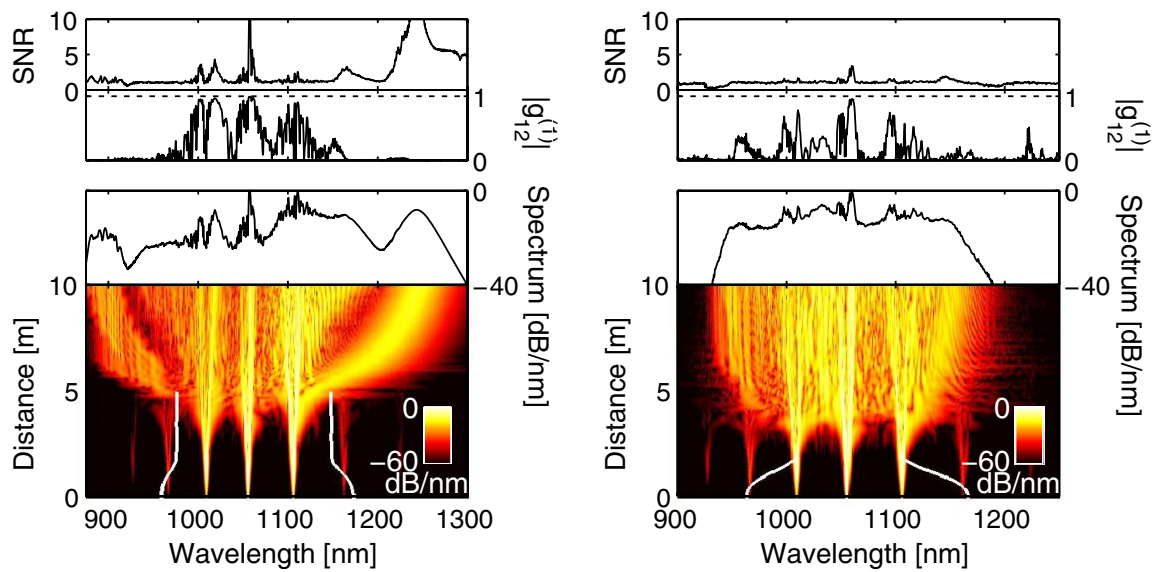

Fig. 3. (Color online) Single-shot simulation (a) with and (b) without the Raman effect. Parameters like in Fig. 2 (c): $1055 \mathrm{~nm}$ pump with $250 \mathrm{~W}$ peak power and a 5\% seed at $13 \mathrm{THz}$. The white lines indicate the MI gain bandwidth. The top rows show the ensemble calculated signal-to-noise ratio (SNR), spectral coherence $\left(\left|g_{12}^{(12)}\right|\right)$, and averaged output spectrum. 
(iv) $\nu_{\mathrm{MI}}<\nu_{\mathrm{mod}}$ : FWM sidebands outside MI gain, no improvement of seeding.

These conclusions will be further specified in the next section in terms of overall coherence and SNR.

\section{B. Overall Statistics}

The ensemble averaged results for both the spectrum, coherence and SNR from Fig. 2 are shown in Fig. 4 for pump-seed frequency offsets spanning well beyond the entire MI gain spectrum. The results in Fig. $\underline{4}$ show an almost perfect symmetry around zero frequency offset, which indicates that seeding the Stokes and anti-Stokes bands yield near identical results. We found that this was a general trend in agreement with the results reported in [24], and we will therefore limit the further analysis to seeding in the Stokes band.

The regimes highlighted in connection with Fig. 2 are easily identified in Fig. 4: For small frequency offsets $(\lesssim 5 \mathrm{THz})$, cascaded FWM with many peaks leads to a coherent pulse breakup and a spectrum with high coherence and SNR. When the frequency offset is increased, the number of amplified peaks decreases and the coherence of the central part of the spectrum degrades. For frequency offsets of $\sim 10-20 \mathrm{THz}$ only a single set of sidebands are amplified via FWM and a large amplitude soliton is generated. The spectrum is only coherent near the residual pump and FWM sidebands, but the soliton is intensity stable. In a frequency range around $\sim 20 \mathrm{THz}$ no large amplitude soliton is ejected, but the FWM sidebands undergo SPM and broaden coherently with a high intensity stability. In this regime, the coherence improves when the frequency offset approaches the MI gain peak. This growth has not been clearly observed before. When the seed is shifted outside the MI gain spectrum, the spectrum is largely incoherent except near the weak FWM sidebands. The coherence plot further shows a high degree of coherence near the higher-order sidebands, but they are too low in amplitude to be visible in the output spectra and will hence only have a minimal effect on the overall coherence.

These general trends are all nicely captured by the overall coherence and SNR. As a rule of thumb for the parameters considered here, regimes of improved coherence can be associated with $\left\langle\left|g_{12}^{(1)}\right|\right\rangle>0.5$. From this criteria the two regimes of improved coherence are easily identified in the overall coherence. The second regime of high coherence is found around 3/4th of the peak of the MI gain of the undepleted pump, which is roughly where the set of FWM sidebands see the highest gain when pump depletion is taken into account.

The spectral coherence is almost mirror symmetric around the pump wavelength, whereas the SNR is asymmetric and generally higher for wavelengths above the pump. This reflects the FWM nature of the seeding that leads to a deterministic and symmetric pulse breakup with high coherence near the FWM bands. The subsequent soliton generation is phasedependent and incoherent, but tends to give a high intensity stability in the wavelength region above the pump. As we shall see, it is actually possible for some seeding conditions to generate the soliton coherently.

\section{Effect of MI Gain Spectrum and Seed Power}

With the general mechanisms dominating seeding at varying pump-seed frequency offsets established, we now turn to investigating the influence of the MI gain spectrum and seed power. Figure $\underline{5}$ shows the overall coherence and SNR as a function of frequency offset for pump wavelengths ranging from 1054.5 to $1075 \mathrm{~nm}$, which gradually decreases the MI gain bandwidth. For all pump wavelengths we show the results for an extensive range of seed peak powers from $0.01 \%$ to $20 \%$ of the pump, and for $5 \%$ we also show the results without the Raman effect [see legend in (f)].

The best coherence improvement is observed for a seed power above $1 \%$ of the pump, which gives sufficient power to initiate the FWM cascade. The intensity improvement is more sensitive to the exact seed power and frequency offset, but is again best for seed powers above $1 \%$ of the pump. The coherence and SNR are generally improved in the long wavelength end of the MI gain spectrum when the power of the seed is increased, because the seed acts like a separate pump that remains (partly) coherent like the pump. The effects of seeding the pulse break-up will be diminished in this regime because of temporal walk-off (see Fig. 1). (a) Spectra

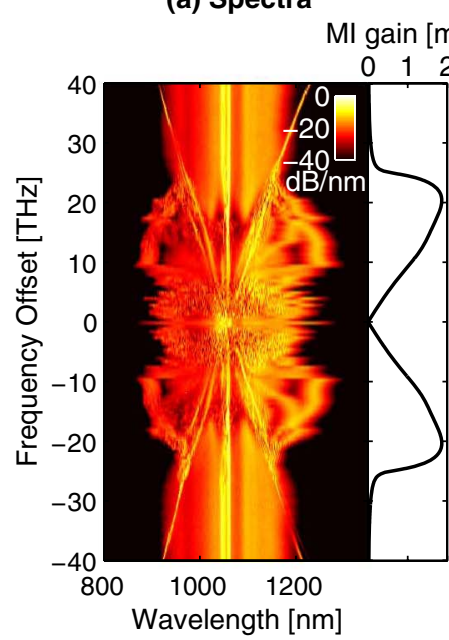

(b) Coherence

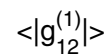

(c) Signal-to-noise ratio

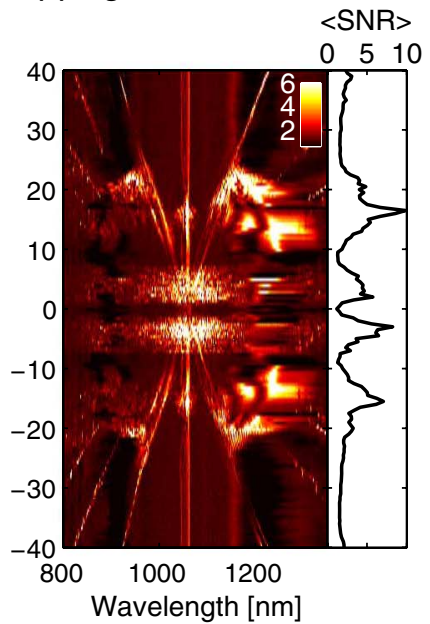

Fig. 4. (Color online) Results of pumping at $1055 \mathrm{~nm}$ with a $5 \%$ seed. Density plots of the (a) output spectral density, (b) coherence, and (c) SNR as a function of wavelength and pump-seed frequency offset. The figures to the right of the density plots show the (a) MI gain, (b) overall coherence, and (c) overall SNR as a function of pump-seed frequency offset. 
(a) $1054.5 \mathrm{~nm}$

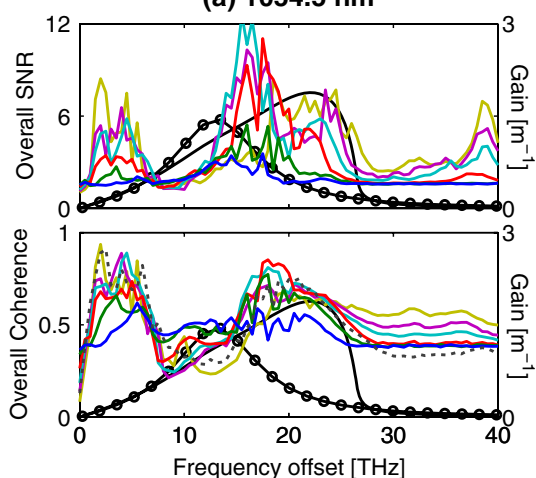

(d) $1057.5 \mathrm{~nm}$

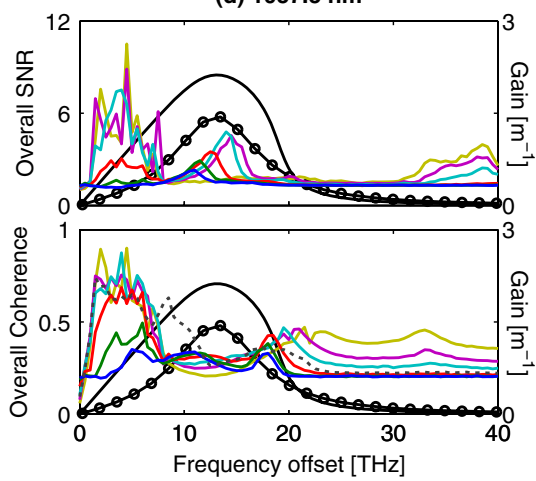

(b) $1055 \mathrm{~nm}$

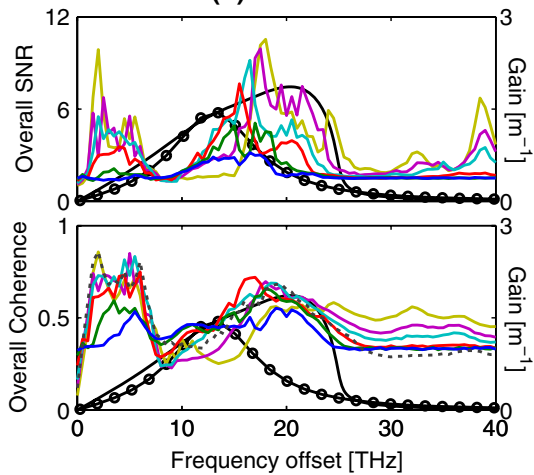

(e) $1064 \mathrm{~nm}$

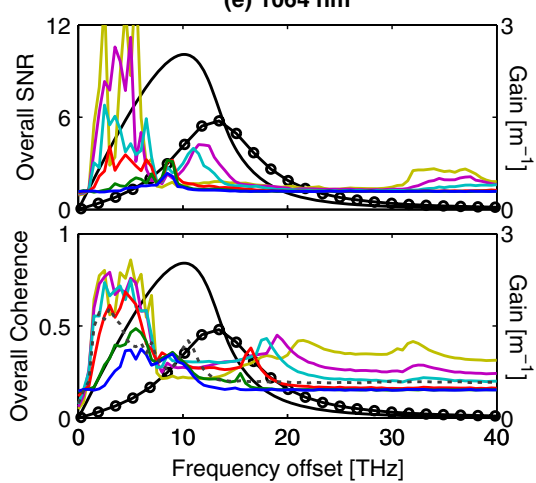

(c) $1056 \mathrm{~nm}$

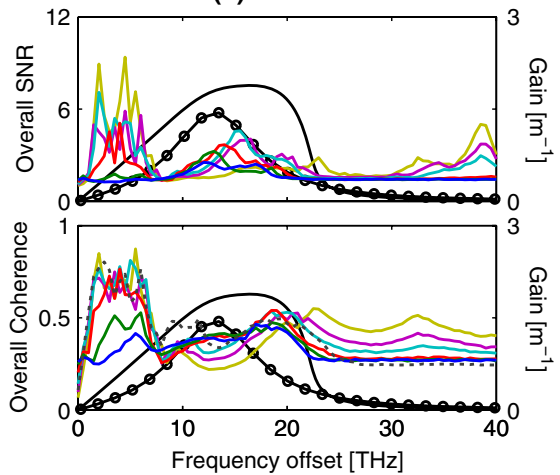

(f) $1075 \mathrm{~nm}$

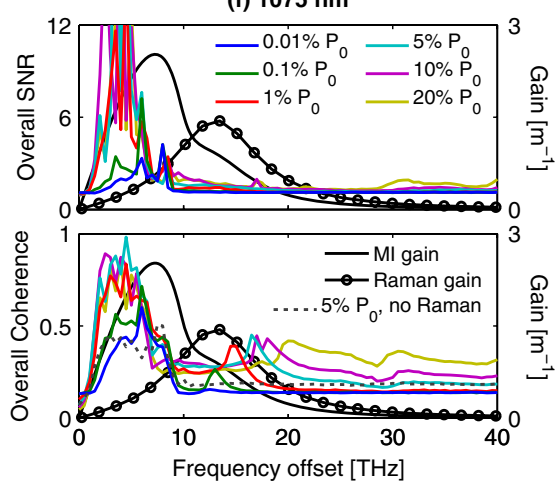

Fig. 5. (Color online) Overall SNR and coherence as a function of pump-seed frequency offset for seed peak powers ranging from $0.01 \%$ to $20 \%$ of the pump peak power, $P_{P}=250 \mathrm{~W}$ [see legend in (f)]. The pump wavelength is (a)-(f) $1054.5,1055,1056,1057.5,1064$, and 1075 nm, respectively, which gradually narrows the MI gain spectrum (full black line). The black circled line shows the Raman spectrum.

Pumping at 1054.5 and $1055 \mathrm{~nm}$ [Figs. $5(\mathrm{a})-5(\mathrm{~b})]$ gives very similar results, and the general trends can be clearly divided into regimes of high and low coherence, as discussed earlier. Interestingly, the overall coherence is almost unaffected when the Raman effect is turned off and the dip from $\sim 8-12 \mathrm{THz}$ remains. This can be explained by the wide MI gain spectrum that is almost uninfluenced by the Raman effect, which gives a smooth gain curve with a sharp cut-off. When the pump is moved further away from the ZDW the MI gain bandwidth decreases and becomes increasingly influenced by the Raman effect, in particular the tail of the Raman gain. In Figs. 5(e) $-5(\mathrm{f})$ the MI gain bandwidth is very narrow, and it is only possible to amplify FWM sidebands that are relatively close to the pump. An overall coherence and stability improvement is therefore only observed for small frequency offsets, i.e., for cascaded FWM with several closely spaced sidebands.

In Figs. $5(\mathrm{a})-5(\mathrm{c})$, the MI gain peak is above the Raman gain peak, and the gain spectrum therefore has a very sharp cut-off due to a minimal contribution from the long-tailed Raman gain. The coherence improvement is consequently nearly the same irrespectively of the Raman effect. In Figs. 5(c)-5(f) the Raman gain has a much stronger effect on the MI gain spectrum that adds a shoulder to the tail of the spectrum, and the coherence improvement is hence decreased without the Raman effect.

The MI gain for small frequency offsets increases significantly when the pump wavelength is increased, which results in higher overall SNR. To further illustrate the difference for small frequency offsets, we show in Fig. 6 the spectral evolution for single simulations for the same pump wavelengths as in Fig. 5 but for a fixed frequency offset of 4 THz. In Figs. $6(\mathrm{a})-\overline{6}$ (d) the pump is very close to the ZDW (black dashed line) and the FWM cascade is slowly amplified and broadened. When the pump is moved further away from the ZDW in Figs. 6(e)-6(f), the MI gain is higher and the FWM cascaded is amplified faster. A higher gain and faster amplification of the FWM cascade diminish the influence of noise.

These results can be divided into two broad regimes depending on the bandwidth of the MI gain, $\nu_{\mathrm{MI}}$, and the peak of the Raman gain, $\nu_{\text {Raman }}$.

(i) $\quad \nu_{\mathrm{MI}} \gg \nu_{\text {Raman }}$ : The MI gain spectrum has a sharp cut-off and the coherence will be improved both for small frequency offsets and in a band near $\nu_{\mathrm{MI}}$.

(ii) $\nu_{\mathrm{MI}} \ll \nu_{\text {Raman }}$ : The MI gain is high for small frequency offsets and has a slowly decreasing tail from the Raman effect. This gives a single region with improved coherence and SNR for small frequency offsets.

The results in [24] correspond to the first case while [20] corresponds to the second.

The overall coherence and SNR in all cases show a strong dependence on the exact frequency offset of the seed. We emphasize that this is not a numerical artefact but reflects that the seeding process is very sensitive to the exact input parameters.

\section{Coherent and Incoherent Rogue Waves}

There is a striking difference in the statistical properties of the large rogue-like solitons generated in Fig. 6: In (b) a powerful soliton is generated completely incoherently, but 
(a) $1054.5 \mathrm{~nm}, 4 \mathrm{THz}$

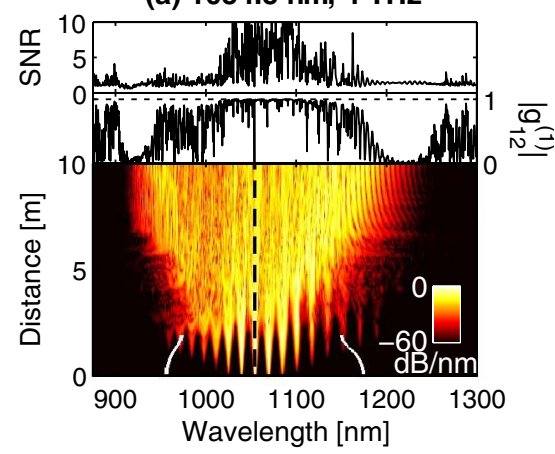

(c) $1056 \mathrm{~nm}, 4 \mathrm{THz}$

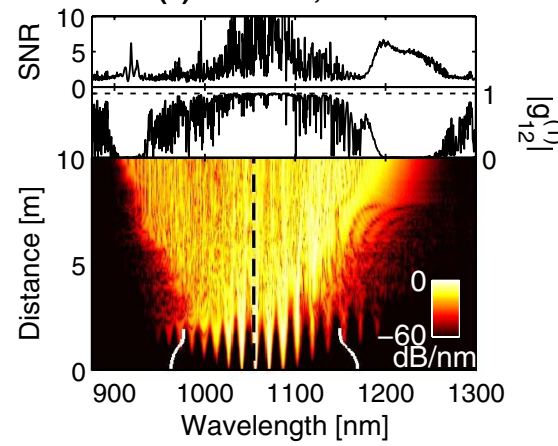

(e) $1064 \mathrm{~nm}, 4 \mathrm{THz}$

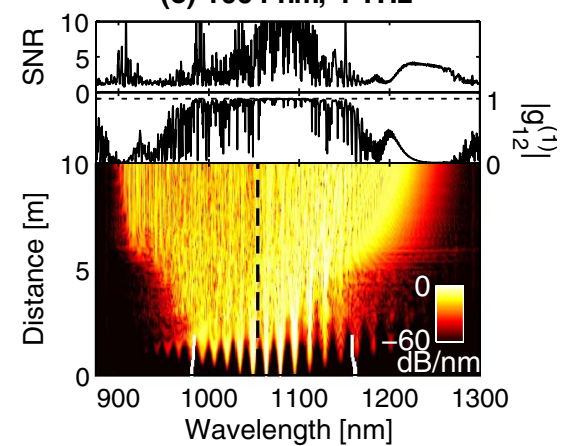

(b) $1055 \mathrm{~nm}, 4 \mathrm{THz}$

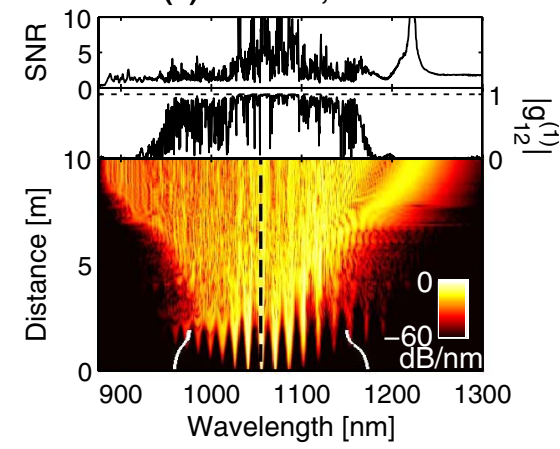

(d) $1057.5 \mathrm{~nm}, 4 \mathrm{THz}$

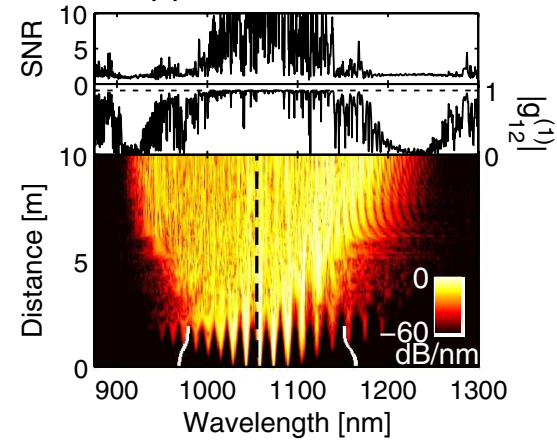

(f) $1075 \mathrm{~nm}, 4 \mathrm{THz}$

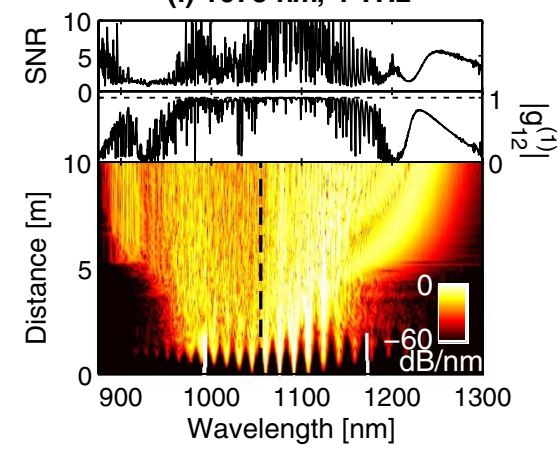

Fig. 6. (Color online) Single-shot simulations of a $5 \%$ seed with a $4 \mathrm{THz}$ offset for the pump wavelengths in Fig. 5 . The white lines indicate the MI gain bandwidth and the black dashed line the ZDW. The top rows show the ensemble calculated SNR and spectral coherence $\left(\left|g_{12}^{(12)}\right|\right)$.

with high SNR, whereas in (f) the rogue soliton is generated with both high coherence and SNR. The existence of such two distinct different types of rogue waves has, to the best of our knowledge, not been demonstrated before.

To understand the difference between a coherent and incoherent rogue wave, we show in Fig. 7 the temporal evolution and spectrogram at the fiber output corresponding to the results in Figs. 6(b) and 6(f). The seed causes a beating of the temporal profile, which leads to a deterministic pulse breakup. When the pump is close to the ZDW [Fig. 7(a)], the MI gain is small and slowly increasing with frequency. The temporal profile is therefore only slowly broken up into solitons. This means that the solitons are mainly generated from the pulse center where the peak power is highest. The solitons have time to redshift before the cascade is amplified and the dynamics is relatively turbulent. In contrast to this, pumping further from the ZDW [Fig. 7(b)] gives a much larger and more rapidly increasing gain. This causes a fast breakup of the temporal pulse, where the individual temporal fringes generate fundamental solitons in a controlled fashion that almost resembles soliton fission. The most powerful solitons are still generated near the center of the pulse where the power is highest. These powerful solitons only collide with the smaller solitons generated from the trailing edge of the pulse. To summarize,

(i) Coherent rogue wave (high coherence and SNR): A large and rapidly increasing MI gain gives a fast breakup of the pulse into solitons. The solitons are generated deterministically with high coherence and are not very affected by collisions.

(ii) Incoherent rogue wave (low coherence, high SNR): A small and slowly increasing gain allows the solitons to start redshifting before the FWM cascade is fully amplified. The coherence is degraded due to collisions.

We emphasize that the generation of both coherent and incoherent rogue solitons was observed for several of the frequency offsets and pump wavelengths considered here.

\section{SEEDING AT HIGH PEAK POWER}

The results presented in the previous sections and in the literature $[19,20,22-24,32]$ all indicate that a cleverly chosen 
(a) $1055 \mathrm{~nm}, 4 \mathrm{THz}$
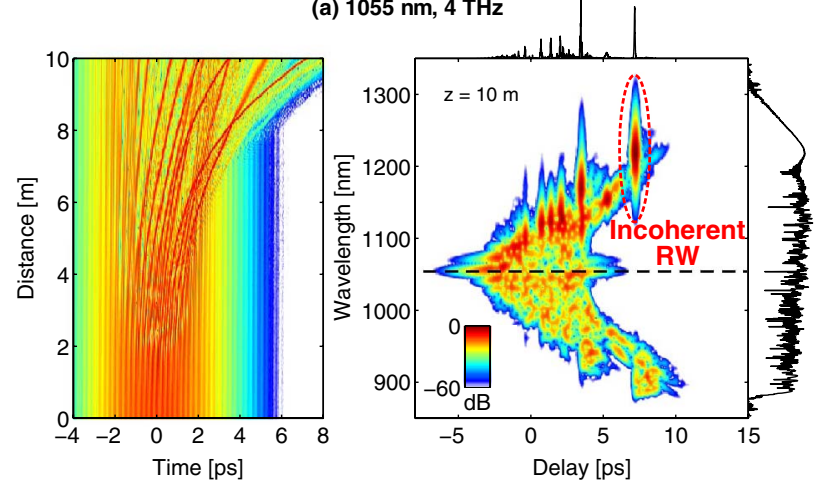

(b) $1075 \mathrm{~nm}, 4 \mathrm{THz}$
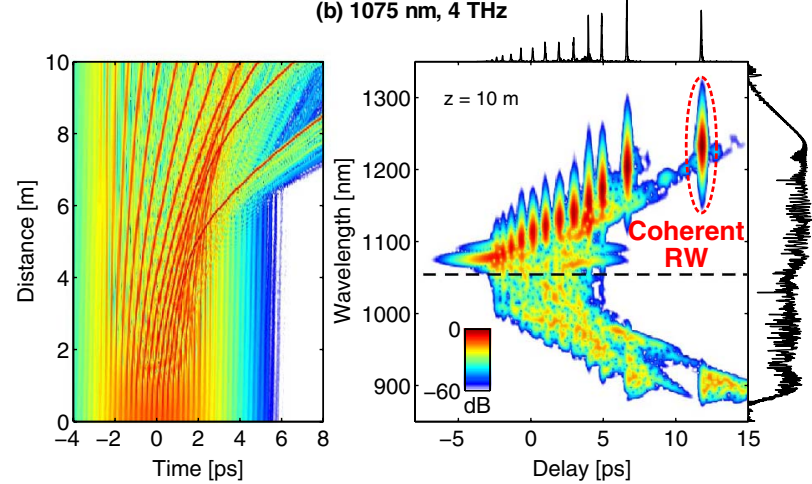

Fig. 7. (Color online) Temporal evolution and spectrogram at the fiber end $(10 \mathrm{~m})$ for a $5 \%$ seed with a $4 \mathrm{THz}$ offset for pump wavelengths of (a) $1055 \mathrm{~nm}$ and (b) $1075 \mathrm{~nm}$, corresponding to Figs. 6(b) and 6(f). The black dashed lines in the spectrograms mark the $\mathrm{Z} \overline{\mathrm{DW}}$. seed can be used to effectively manipulate the pulse breakup and improve the noise characteristics or even trigger the SC generation. While it has thus been demonstrated that seeding a low peak power pump offers several improvements, the situation is very different when the peak power of the pump is increased, as we shall now demonstrate. The coherence properties at high power were discussed in [21], but only over very short propagation distances where the spectrum remains narrow.

Figure 8 shows the spectral evolution of single shot simulations when pumping at the Ytterbium wavelength of $1064 \mathrm{~nm}$ with a $5 \%$ seed at $3 \mathrm{THz}$ offset for pump peak powers of 500 , 750 , and $1500 \mathrm{~W}$, respectively. For a $250 \mathrm{~W}$ pump the $3 \mathrm{THz}$ frequency offset gave the best coherence improvement [see Fig. 5(e)], due to the controlled breakup of the pump by cascaded FWM, resulting in an overall coherence of 0.66 . In Fig. 8(a) the peak power is doubled to $500 \mathrm{~W}$. The increased pump power results in a wider spectrum, but the overall coherence is reduced to 0.33 and the coherence improvement is limited to the central part of the spectrum that was directly generated by the initial cascaded FWM. The peak power is increased to $750 \mathrm{~W}$ in Fig. 8(b), which leads to the generation of several distinct solitons and GV matched DWs. The spectrum is not nearly as coherent as Fig. 8(a): The overall coherence is 0.16 and the coherence improvement is again limited to the central part of the spectrum. When the peak power is increased to $1500 \mathrm{~W}$ in Fig. 8(c), the initial FWM cascade is quickly washed out by the onset of highly phase-dependent soliton interaction and DW generation, and the output spectrum is incoherent over nearly the entire bandwidth with (a) $500 \mathrm{~W}$

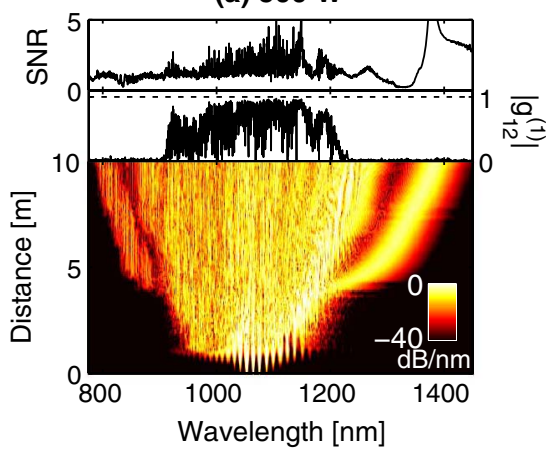

(c) $1500 \mathrm{~W}$

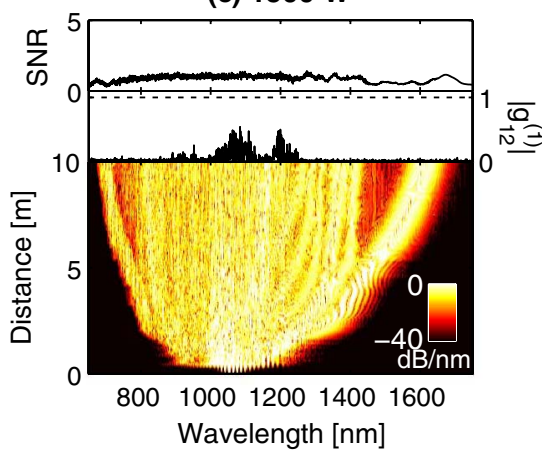

(b) $750 \mathrm{~W}$

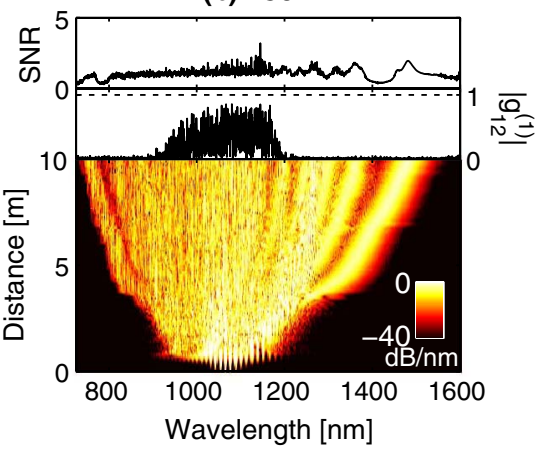

(d) $\quad-500 \mathrm{~W} \quad \cdots 1500 \mathrm{~W}$

$--750 \mathrm{~W} \rightarrow$ Raman gain

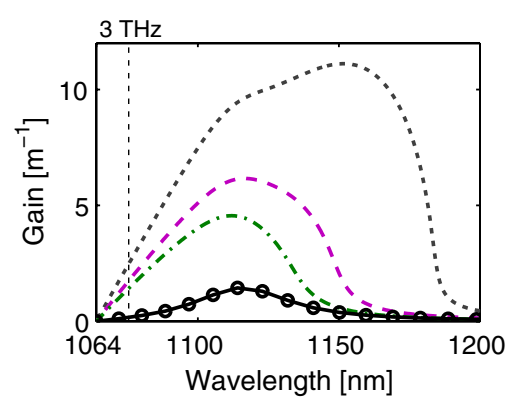

Fig. 8. (Color online) Single-shot simulations of pumping at $1064 \mathrm{~nm}$ with a $5 \%$ seed at a frequency offset of $3 \mathrm{THz}$ for pump peak powers of (a)-(c) 500, 750, and $1500 \mathrm{~W}$, respectively. The top rows show the signal-to-noise ratio (SNR) and spectral coherence $\left(\left|g_{12}^{(12)}\right|\right)$. (d) MI and Raman gain spectra. The vertical line marks $3 \mathrm{THz}$ offset. 
(a) $500 \mathrm{~W}$
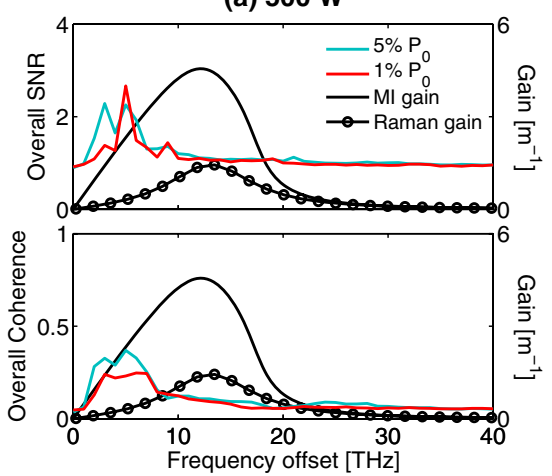

(b) $750 \mathrm{~W}$

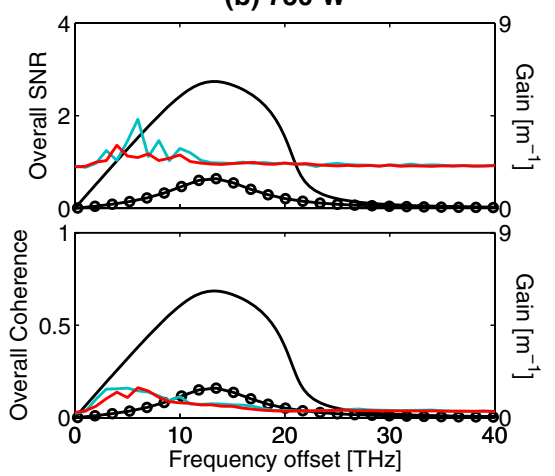

(c) $1500 \mathrm{~W}$

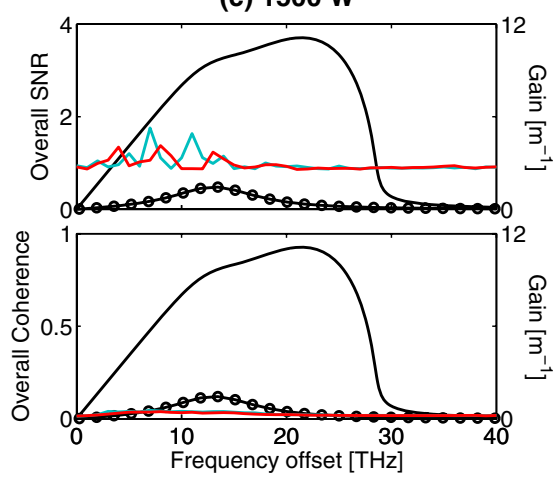

Fig. 9. (Color online) Overall SNR and coherence as a function of pump-seed frequency offset, shown for seed peak powers of $1 \%$ and $5 \%$ of the pump peak power [see legend in (a)]. The pump wavelength was $1064 \mathrm{~nm}$ and the peak power (a)-(c) 500, 700, and 1500 W. The MI and Raman spectra are shown with the full and circled black lines, respectively.

an overall coherence of just 0.040 . In all cases, the formation of rouge-like solitons leads to small improvements in the intensity stability around the soliton, but this too seems to be gradually washed out by the turbulent dynamics that govern the evolution and interaction of many solitons and DWs when the peak power is increased.

In Fig. 9 is shown the overall coherence and SNR corresponding to peak powers of 500, 750, and $1500 \mathrm{~W}$, which are the same peak powers that were used in Fig. 8. The results for the lowest peak power in Fig. 9(a) show a minor improvement for seeding close to the pump, but nothing like what was observed in Fig. 5(e) at $250 \mathrm{~W}$. When the peak power is increased further in Figs. 9(b) -9 (c), there is basically no overall improvement in coherence and intensity stability irrespectively of the pump-seed frequency offset.

When the pump power is increased the improvements afforded by seeding are thus quickly washed out by turbulent solitonic dynamics. In [10] it was experimentally demonstrated that the spectral noise increases with the pump power, although this was for a very different set-up where a fiber with two closely spaced ZDWs was back-seeded. As discussed in [21], the pulse breakup can be completely deterministic and coherent also at high pump powers, but as soon as the initial comb structure is broken into solitons the coherence is degraded by the subsequent highly phase-dependent interactions. In other words, the typical broadband and flat SC spectrum in most high-power experiments comes at a price of a low coherence as it is intrinsically dominated by solitonic dynamics.

\section{DISCUSSION AND CONCLUSIONS}

It seems doubtful that seeding can improve the noise properties at power levels like those in commercial SC sources. Although a coherent pulse breakup can be achieved at these power levels, the coherence will only be preserved over a very short propagation distance for which the spectrum remains relatively narrow. Seeding nonetheless remains an interesting approach for optical switching [19], where the presence of a seed pulse triggers the SC broadening that would otherwise be below threshold.

Seeding is very sensitive to the exact input parameters, such as the wavelength and power of both the pump and seed, and ultimately has to be studied case-by-case. It is however possible to identify some broad regimes dominated by certain mechanisms, as demonstrated in this work. Controlling the generation of rogue waves by modulating the pump pulse therefore also seems limited to relatively low power levels. In particular, it is difficult to imagine that extremely large solitonic rogue waves can be deterministically generated by seeding a high power pump, as these waves can only be generated through energy transfer from many collisions, which cannot be controlled by seeding.

In conclusion, we have investigated the influence of the pump power and MI gain spectrum on seeding. We analyzed the results both in terms of spectral coherence and intensity stability. For a low pump power we found that seeding can give a deterministic pulse breakup due to FWM between the seed and pump. The overall stability of the spectrum can be improved by seeding close to the pump, which gives a broad FWM cascade with many sidebands. However, for a broad MI spectrum, an overall stability improvement is also observed when seeding close to the peak of the MI spectrum, which allows a single set of FWM sidebands to be coherently amplified. It was demonstrated that rogue waves can be excited both coherently and incoherently from the FWM cascade, depending on the MI gain. Finally, it was found that seeding has no or little influence on the noise properties when the pump power is increased to the $\mathrm{kW}$ level. At these power levels turbulent solitonic dynamics quickly washes out the coherent pulse breakup.

\section{ACKNLOWLEDGMENT}

The authors thank the Danish Agency of Science, Technology and Innovation for support of the project no. 09-070566.

\section{REFERENCES}

1. J. M. Dudley, G. Genty, and S. Coen, "Supercontinuum generation in photonic crystal fiber," Rev. Mod. Phys. 78, 1135-1184 (2006).

2. P. Beaud, W. Hodel, B. Zysset, and H. Weber, "Ultrashort pulse propagation, pulse breakup, and fundamental soliton formation in a single-mode optical fiber," IEEE J. Quantum Electron. 23, 1938-1946 (1987).

3. J. M. Stone and J. C. Knight, "Visibly 'white' light generation in uniformphotonic crystal fiber using a microchip laser," Opt. Express 16, 2670-2675 (2008).

4. J. C. Travers and J. R. Taylor, "Soliton trapping of dispersive waves in tapered optical fibers," Opt. Lett. 34, 115-117 (2009).

5. J. C. Travers, "Blue extension of optical fibre supercontinuum generation," J. Opt. 12, 113001 (2010). 
6. S. T. Sørensen, A. Judge, C. L. Thomsen, and O. Bang, "Optimum fiber tapers for increasing the power in the blue edge of a supercontinuum-group-acceleration matching," Opt. Lett. 36, 816-818 (2011).

7. M. H. Frosz, P. M. Moselund, P. D. Rasmussen, C. L. Thomsen, and O. Bang, "Increasing the blue-shift of a supercontinuum by modifying the fiber glass composition," Opt. Express 16, 21076-21086 (2008).

8. V. Tombelaine, C. Lesvigne, P. Leproux, L. Grossard, V. Couderc, J.-L. Auguste, J.-M. Blondy, G. Huss, and P.-H. Pioger, "Ultra wide band supercontinuum generation in air-silica holey fibers by SHG-induced modulation instabilities," Opt. Express 13, 7399-7404 (2005).

9. J. C. Travers, S. V. Popov, and J. R. Taylor, "Extended blue supercontinuum generation in cascaded holey fibers," Opt. Lett. 30, 3132-3134 (2005).

10. P. M. Moselund, M. H. Frosz, C. L. Thomsen, and O. Bang, "Backseeding of higher order gain processes in picosecond supercontinuum generation,” Opt. Express 16, 11954-11968 (2008).

11. P. M. Moselund, "Long-pulsed supercontinuum sources," Ph.D. dissertation, DTU Fotonik, Dept. of Photonics Engineering, Technical Univ. of Denmark (2009).

12. N. Brauckmann, M. Kues, T. Walbaum, P. Groß, and C. Fallnich, "Experimental investigations on nonlinear dynamics in supercontinuum generation with feedback," Opt. Express 18, 7190-7202 (2010).

13. M. N. Islam, G. Sucha, I. Bar-Joseph, M. Wegener, J. P. Gordon, and D. S. Chemla, "Femtosecond distributed soliton spectrum in fibers,” J. Opt. Soc. Am. B 6, 1149-1158 (1989).

14. O. Bang and M. Peyrard, "Generation of high-energy localized vibrational modes in nonlinear Klein-Gordon lattices," Phys. Rev. E 53, 4143-4152 (1996).

15. O. Bang and P. D. Miller, "Exploiting discreteness for switching in waveguide arrays," Opt. Lett. 21, 1105-1107 (1996).

16. F. Luan, D. V. Skryabin, A. V. Yulin, and J. C. Knight, "Energy exchange between colliding solitons in photonic crystal fibers," Opt. Express 14, 9844-9853 (2006).

17. G. Genty, C. M. de Sterke, O. Bang, F. Dias, N. Akhmediev, and J. M. Dudley, "Collisions and turbulence in optical rogue wave formation,” Phys. Lett. A 374, 989-996 (2010).

18. D. R. Solli, C. Ropers, P. Koonath, and B. Jalali, "Optical rogue waves," Nature 450, 1054-1057 (2007).
19. D. R. Solli, C. Ropers, and B. Jalali, "Active control of rogue waves for stimulated supercontinuum generation,” Phys. Rev. Lett. 101, 233902 (2008).

20. G. Genty, J. Dudley, and B. Eggleton, "Modulation control and spectral shaping of optical fiber supercontinuum generation in the picosecond regime," Appl. Phys. B 94, 187-194 (2009).

21. G. Genty and J. Dudley, "Route to coherent supercontinuum generation in the long pulse regime," IEEE J. Quantum Electron. 45, 1331-1335 (2009).

22. D. R. Solli, B. Jalali, and C. Ropers, "Seeded supercontinuum generation with optical parametric down-conversion," Phys. Rev. Lett. 105, 233902 (2010).

23. K. K. Y. Cheung, C. Zhang, Y. Zhou, K. K. Y. Wong, and K. K. Tsia, "Manipulating supercontinuum generation by minute continuous wave," Opt. Lett. 36, 160-162 (2011).

24. Q. Li, F. Li, K. K. Y. Wong, A. P. T. Lau, K. K. Tsia, and P. K. A Wai, "Investigating the influence of a weak continuous-wavetrigger on picosecond supercontinuum generation," Opt. Express 19, 13757-13769 (2011).

25. M. H. Frosz, T. Sørensen, and O. Bang, "Nanoengineering of photonic crystal fibers for supercontinuum spectral shaping," J. Opt. Soc. Am. B 23, 1692-1699 (2006).

26. G. Agrawal, Nonlinear Fiber Optics, 4th ed. (Academic, 2007)

27. C. Agger, C. Petersen, S. Dupont, H. Steffensen, J. K. Lyngs $\varnothing$, C. L. Thomsen, J. Thøgersen, S. R. Keiding, and O. Bang, "Supercontinuum generation in ZBLAN fibers--detailed comparison between measurement and simulation," J. Opt. Soc. Am. B 29, 635-645 (2012).

28. J. Laegsgaard, "Mode profile dispersion in the generalised nonlinear Schrödinger equation,” Opt. Express 15, 16110-16123 (2007).

29. J. Hult, "A fourth-order Runge-Kutta in the interaction picture method for simulating supercontinuum generation in optical fibers," J. Lightwave Technol. 25, 3770-3775 (2007).

30. J. M. Dudley and S. Coen, "Coherence properties of supercontinuum spectra generated in photonic crystal and tapered optical fibers," Opt. Lett. 27, 1180-1182 (2002).

31. S. T. Sørensen, O. Bang, B. Wetzel, and J. M. Dudley, "Describing supercontinuum noise and rogue wave statistics using higher-order moments," Opt. Commun. 285, 2451-2455 (2012).

32. J. M. Dudley, G. Genty, and B. J. Eggleton, "Harnessing and control of optical rogue waves in supercontinuum generation," Opt. Express 16, 3644-3651 (2008). 